\title{
Measurement of the cross section for prompt isolated diphoton production at the Tevatron
}

\author{
Costas Vellidis ${ }^{* \dagger}$ \\ FNAL \\ E-mail: vellidis@fnal.gov
}

This article reports a measurement of the cross section of prompt isolated photon pair production in $p \bar{p}$ collisions at a total energy $\sqrt{s}=1.96 \mathrm{TeV}$ using data of $5.36 \mathrm{fb}^{-1}$ integrated luminosity collected with the CDF II detector at the Fermilab Tevatron. The measured cross section, differential in basic kinematic variables, is compared with three perturbative QCD predictions, a leading order (LO) parton shower calculation and two next-to-leading order (NLO) calculations. The NLO calculations reproduce most aspects of the data. By including photon radiation from quarks before and after hard scattering, the parton shower prediction becomes competitive with the NLO predictions.

XXIst International Europhysics Conference on High Energy Physics

Grenoble, Rhône-Alpes France

\footnotetext{
${ }^{*}$ Speaker.

${ }^{\dagger}$ On behalf of the CDF Collaboration.
} 
The measurement of the production cross section of two energetic isolated central photons (diphotons) in high energy hadron collisions is important for testing standard model predictions in the domain of searches for undiscovered particles and new physics. The reported measurement [1] was conducted using data of total integrated luminosity $5.36 \mathrm{fb}^{-1}$ collected with the Collider Detector at Fermilab (CDF) at the Tevatron $p \bar{p}$ collider. The background from $\gamma+$ jet and dijet events, where one or two jets are faking a photon, is subtracted using the track isolation as the discriminant between signal and background.

The results of this measurement are compared with three calculations: (i) the fixed NLO predictions of the DIPHOX program including parton fragmentations into photons, (ii) the predictions of the RESBOS program where the cross section is accurate to NLO, but also has an analytical initial state soft gluon resummation with a next-to-next-to-leading-log (NNLL) accuracy, and (iii) the predictions of the PYTHIA program which features a realistic representation of the physics events by including parton showering, initial (ISR) and final state radiation (FSR) and an underlying event

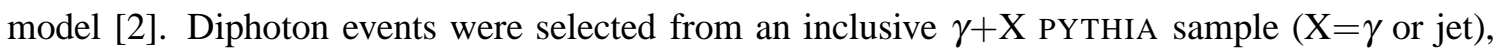
thus including the $q \bar{q} \rightarrow \gamma \gamma$ and $g g \rightarrow \gamma \gamma$ processes (56\%) as well as the $q \bar{q} \rightarrow g \gamma \gamma_{\text {ISR }}, g q \rightarrow q \gamma \gamma_{\text {ISR }}$ and $g q \rightarrow q \gamma \gamma_{\mathrm{FSR}}$ processes (44\%). This type of calculation effectively resums the cross section for gluon and photon radiation both in the initial and the final state with a leading-log (LL) accuracy.

Fig. 1 shows the comparison between the measured and predicted diphoton cross sections as functions of the diphoton invariant mass $M$, the diphoton transverse momentum $P_{\mathrm{T}}$ and the difference $\Delta \phi$ between the azimuthal angles of the two photons in the event. The shaded area around the data points indicates the total systematic uncertainty of the measurement. While the PYTHIA direct calculation $(\gamma \gamma)$ fails to describe both the scale and shape of the data, including radiation brings the prediction in fair agreement with the data. Discrepancies between data and theory are observed in the mass spectrum below the peak at $30 \mathrm{GeV} / \mathrm{c}^{2}$, in the $P_{\mathrm{T}}$ spectrum between 20 and $50 \mathrm{GeV} / \mathrm{c}$, and most prominently in the $\Delta \phi$ spectrum. For $\Delta \phi>2.2 \mathrm{rad}$, where soft gluon processes are expected to manifest, the RESBOS prediction agrees better with the data. In the range $1.4 \mathrm{rad}<\Delta \phi<2.2 \mathrm{rad}$ only the PYTHIA $\gamma \gamma+\gamma \mathrm{j}$ prediction describes the data and remains closest to the data down to $1 \mathrm{rad}$. For $\Delta \phi<1 \mathrm{rad}$ all three predictions are lower than the data, although the DIPHOX prediction, by explictly including non-perturbative fragmentation, lies closer to the data.

In summary, the diphoton production cross section, differential in kinematic variables sensitive to the reaction mechanism, is measured using data corresponding to an integrated luminosity of $5.36 \mathrm{fb}^{-1}$ collected with the CDF II detector. The results of the measurement are compared with three state-of-the-art calculations, applying complementary techniques in describing the reaction. All three calculations, within their known limitations, reproduce the main features of the data, but none of them describes all aspects of the data. The inclusion of photon radiation in the initial and final states significantly improves the PYTHIA parton shower calculation.

\section{References}

[1] T. Aaltonen et al. (CDF Collaboration), Phys. Rev. Lett. 107, 102003 (2011); T. Aaltonen et al. (CDF Collaboration), Phys. Rev. D 84, 5, 052006 (2011).

[2] Diphox: T. Binoth et al., Phys. Rev. D63, 114016 (2001); Resbos: C. Balazs et al., Phys. Rev. D76, 013009 (2007); PYTHIA: S. Mrenna, Comput. Phys. Commun. 101, 232 (1997). 

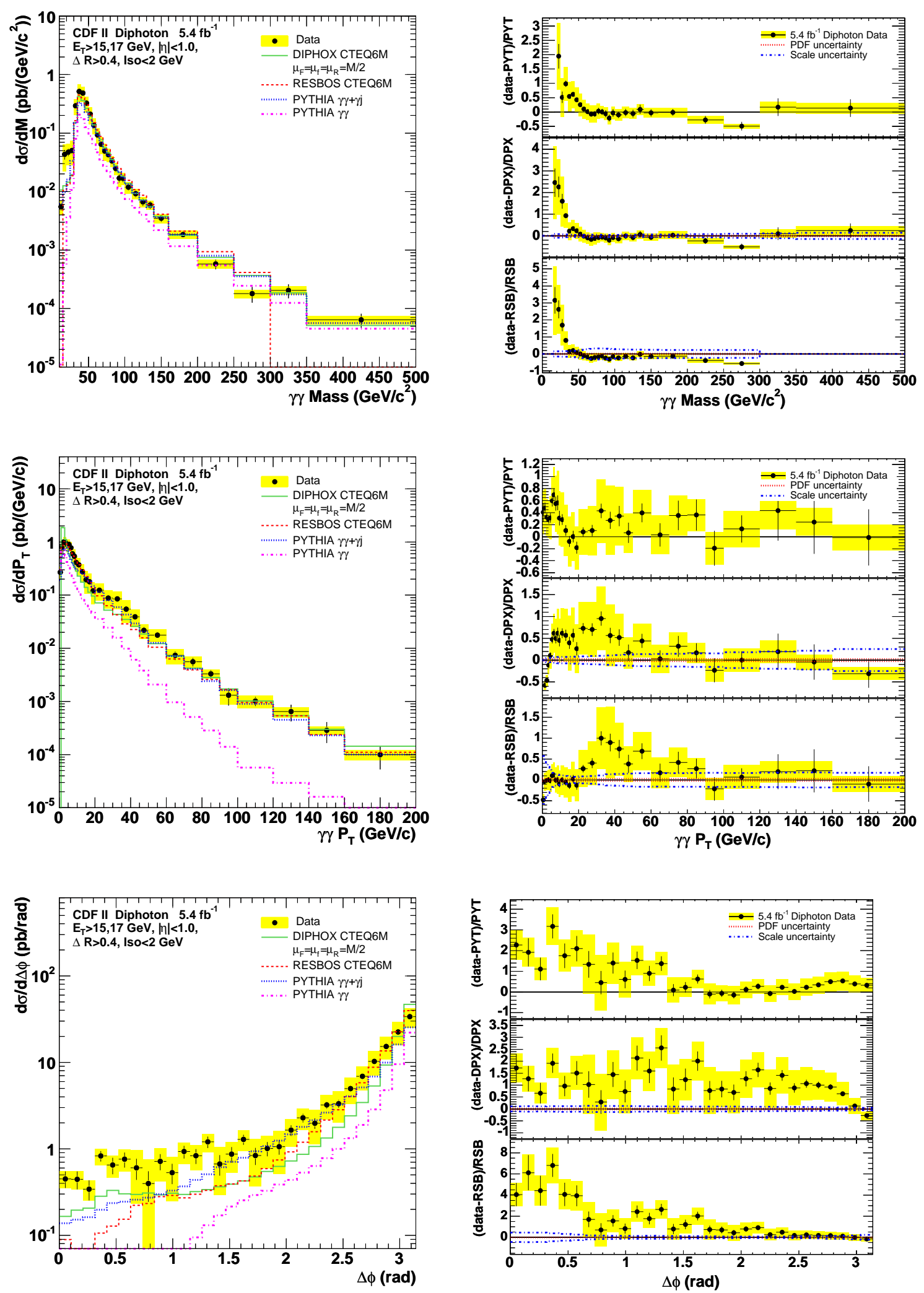

Figure 1: The measured differential cross sections compared with three theoretical predictions discussed in the text. Left: absolute comparisons. Right: fractional deviations of the data from the predictions. Fractional deviations for PYTHIA refer to the $\gamma \gamma+\gamma \mathrm{j}$ calculation. 